\title{
Salivary testosterone responses to a physical and psychological stimulus and subsequent effects on physical performance in healthy adults
}

\author{
Blair T. Crewther, ${ }^{1,2,3}$ Liam P. Kilduff, ${ }^{4}$ Charlie Finn, ${ }^{4,5}$ Phil Scott, ${ }^{6}$ Christian J. Cook ${ }^{2,3,4}$
}

${ }^{1}$ Institute of Sport - National Research Institute, Warsaw, Poland; ${ }^{2}$ Hamlyn Centre, Imperial College, London, ${ }^{3}$ School of Sport Health and Exercise Science Bangor University, Bangor, Wales, ${ }^{4}$ Applied Sports, Technology, Exercise and Medicine (A-STEM) research centre, School of Engineering, Swansea University, Swansea, ${ }^{5}$ University of the West of Scotland, Paisley, UK, ${ }^{6}$ England Team Strength and Conditioning Coach, England and Wales Cricket Board, UK; ${ }^{7}$ Queensland Academy of Sport's Centre of Excellence for Applied Sport Science Research, Queensland, Australia

\begin{abstract}
OBJECTIVE: To address the rapid influence of testosterone (T) on neuromuscular performance, we compared the $T$ and physical performance responses of adults exposed to a physical and psychological stimulus. DESIGN: A group of healthy men $(n=12)$ and women $(n=14)$ each completed three treatments using a randomised, crossover design: exercise involving five $\times$ ten-second cycle sprints, viewing a video clip with aggressive content and a control session. Salivary $T$ concentrations, hand-grip strength (HGS) and countermovement jump peak power (CMJ PP) were assessed before and 15 minutes after each session. RESULTS: The relative changes in T (17 $\pm 29 \%)$ and CMJ PP (-0.1 $\pm 4.4 \%)$ following sprint exercise were superior to the aggressive video $(-6.3 \pm 19 \%,-2.2 \pm 5.9 \%)$ and control $(-4.8 \pm 23 \%,-2.8 \pm 4.4 \%)$ treatments, respectively $(p \leq 0.05)$. Pre-treatment $T$ levels correlated $(r=-0.58$ to $-0.61, p<0.05)$ with the $T$ responses of men (sprint exercise) and women (sprint exercise, aggressive video), but no variables were significantly correlated with the relative changes in HGS or CMJ PP. CONCLUSIONS: Sprint exercise promoted a general rise in $\mathrm{T}$ and maintained CMJ PP, relative to the video and control treatments. In both sexes, those individuals with higher pre-test $T$ levels tended to produce smaller $T$ responses to one or more treatments. These data highlight the importance of stimulus selection and individual predispositions when attempting to acutely modify $T$ and associated physical performance.
\end{abstract}

Key words: Exercise, Gender, Gonadal, Neuromuscular, Priming

Address for correspondence:

Dr. Blair Crewther, Department of Endocrinology,

Instituteof Sport, 01-982 Warsaw, Poland;

E-mail: blair.crewther@gmail.com

Received: 15-12-2015, Accepted: 12-05-2016

\section{INTRODUCTION}

Considerable research has examined the steroid testosterone $(\mathrm{T})$ and its role in mediating athletic performance and training adaptation (see reviews). ${ }^{1,2}$ The traditional viewpoint is that $\mathrm{T}$ helps to control 
muscle size and subsequent force development, ${ }^{1,2}$ but it now appears that transient physiological elevations in $\mathrm{T}$ concentrations are less important to protein metabolism and muscle growth than previously thought. ${ }^{3}$ Still, T might contribute to, or support, many other neuromuscular (e.g. motor system drive, calcium signalling, muscle properties) and psychological (e.g. mood, cognition) functions ${ }^{1}$ to regulate athletic performance on a rapid timescale. Consequently, there is much interest in developing practical strategies to acutely modify $\mathrm{T}$ availability and thereafter physical performance. ${ }^{4}$

Physical exercise provides an effective tool for enhancing $T$ release and indices of muscle performance ${ }^{5-8}$ For example, a morning exercise bout (i.e. sprints, weight training) improved afternoon $\mathrm{T}$ secretion and the physical abilities of male athletes. ${ }^{6}$ Similarly, better training outcomes were achieved by combining arm and leg exercises to elevate physiological hormones (including $\mathrm{T}$ ) during repeated workouts, ${ }^{7,8}$ and by prescribing resistance-exercise workouts that maximised the $\mathrm{T}$ responses of individual athletes. ${ }^{9}$ Testosterone is also responsive to high-intensity sprint cycling (e.g. $2 \times 10$ seconds, $1 \times 40$ seconds), with increases of $9-28 \%$ demonstrated in both trained and untrained male populations. ${ }^{5,10-12}$ Moreover, the T changes occurring are often accompanied by rapid improvements in physical strength ${ }^{5}$ and power. ${ }^{11}$ Thus, this type of exercise could provide a brief, practical and effective stimulus for improving these outcomes.

Psychological stimuli in the form of video footage provides another practical tool for stimulating $\mathrm{T}$ release in men $^{10,13-15}$ with added links to physical performance. ${ }^{16}$ In the latter work, the watching of short video clips (i.e. aggressive, humorous, motivational, erotic) elevated $\mathrm{T}$ levels and improved subsequent voluntary strength in professional athletes. ${ }^{16}$ In addition, the individual changes in $\mathrm{T}$ and strength were strongly related $(\mathrm{r}=0.85)$ across all video clips. ${ }^{16}$ The aggressive video provided the most effective stimulus for achievement of these outcomes. In healthy men (non-athletes), exposure to the same type of video also produced a marginal $\mathrm{T}$ increase of $8-12 \%,{ }^{10,17}$ as did an aggressive video game. ${ }^{18}$ To our knowledge, no studies have examined whether an aggressive video can improve $\mathrm{T}$ availability and physical performance in healthy adults, nor how this treatment compares to a physical stimulus.
Similarly to men, acute elevations in T concentrations have been demonstrated in women undertaking different forms of exercise (e.g. resistance, intermittent and prolonged cycling $)^{19-21}$ and when viewing selected video images (e.g. violence, attractive men). ${ }^{15,22}$ In fact, women can exhibit similar relative changes in $\mathrm{T}$ concentrations to men when exposed to a physical and/or psychological challenge. ${ }^{15,23}$ This means that both sexes can access similar relative amounts of $T$ with an appropriate stimulus, despite women having less than half the amount of $\mathrm{T}$ in circulation. ${ }^{23}$ It has yet to be established whether such changes in the hormonal milieu might produce a comparable performance effect in women, as seen in male populations. This could be addressed by comparing the acute $\mathrm{T}$ responses of healthy men and women to a physical and psychological stimulus.

This study examined the effects of a physical (sprint exercise) and psychological (aggressive video) stimulus on salivary $\mathrm{T}$ concentrations and physical performance (strength, power) in healthy men and women. The first hypothesis was that each stimulus would lead to elevated $\mathrm{T}$ responses and improvements in strength and power in both groups. We also hypothesised that sprint exercise would promote greater $\mathrm{T}$ increases and thus larger performance gains.

\section{METHODS}

\section{Subjects}

Twelve men (mean \pm SD age $24.5 \pm 5.3$ years, height $181.1 \pm 4.8 \mathrm{~cm}$, body mass $83.5 \pm 7.7 \mathrm{~kg}$ ) and 14 women (mean \pm SD age $23.5 \pm 3.9$ years, height $164.4 \pm 5.8$ $\mathrm{cm}$, body mass $59.3 \pm 6.1 \mathrm{~kg}$ ) were recruited from a University campus. Both groups were of similar age ( $p=0.776$ ), but the men were taller and heavier than the women $(p<0.001)$. The participants were classified as recreationally active, based on self-reported activity in the last 12 months (i.e. regular exercise or leisure activities at least 3 times weekly, at least 60 minutes in duration), with no health or medical issues that could influence the study outcomes. Four women did report oral contraceptive use, but we found no differences in baseline $T$ levels, $T$ reactivity or physical performance between this sub-group and the naturally-cycling women, so all data were combined for analysis. We anticipated that the menstrual cycle 
would not influence baseline T or performance. ${ }^{21}$, ${ }^{24}$ Informed consent was signed before the study commenced and ethical approval was provided by the Swansea University Human Ethics Committee, Swansea, UK.

\section{Study design}

The participants each completed three treatments using a randomised, crossover design: exercise involving cycle sprints, viewing an aggressive video and a control session. Salivary T concentrations, hand-grip strength (HGS) and countermovement jump peak power (CMJ PP) were measured before and after each treatment. Before testing, the participants sat quietly for ten minutes in a stress-free environment. ${ }^{10}$ Each session lasted $\sim 48$ minutes and was separated by at least three days recovery, but no more than ten days. All treatments were conducted between 1100 and 1600 hours to address diurnal variation in $\mathrm{T}$ production and T reactivity to physical or psychological stress. ${ }^{25,26}$ Within this timeframe, each participant completed his or her own sessions within a shorter ( \pm 1 hour) period. The participants were told to refrain from all exercise in the preceding 24 hours to eliminate the effects of muscle fatigue. A familiarisation session was also completed to ensure that the participants were capable of completing the sprints and to account for a pronounced stress response due to intense, unaccustomed exercise. ${ }^{10}$

\section{Sprint exercise treatment}

Sprint exercise was performed on a friction-braked cycle ergometer (Monark 824E, Sweden) using a resistance load that equated to $7.5 \%$ of individual body mass. ${ }^{27}$ The men, due to their larger body mass, were prescribed a heavier mean load $(5.8 \pm 0.5 \mathrm{~kg})$ than women $(4.1 \pm 0.4 \mathrm{~kg})$. After a standard warm-up, comprising of two minutes cycling with a light $(1 \mathrm{~kg})$ load, the participants cycled with maximum effort for a period of ten seconds at the pre-selected load. This effort was followed by 50 seconds of slow pedalling without load. The entire sequence was repeated four times, so that five trials were completed over a fiveminute period. The lead investigator provided a three second count down before each sprint trial started and ended. The participants pedalled slowly for a further minute after the last sprint, before sitting quietly in the laboratory. The chosen format was based on prior work to ensure a T rise. ${ }^{5,12,18}$ Toe clips were used to prevent slippage from the pedals and strong verbal encouragement was given to ensure maximal effort was applied during each trial.

\section{Video treatment}

The participants sat by themselves in a separate room to watch the video presentation on a single laptop computer (Dell Inspiron, 15.6 inch screen). The video contained aggressive content of big tackles and collisions in the sport of rugby union, which has been shown to promote small to marginal $\mathrm{T}$ increases in men. ${ }^{10,16,17}$ The video was taken from an online resource (https://www.youtube.com/watch?v=UAaxeTowTd0) and played for five minutes to equalise the duration of stimulus exposure with sprint exercise. The video was accompanied by a somewhat "aggressive" music track to increase the likelihood of a behavioural and thus, hormonal response. ${ }^{28}$ To eradicate other environmental or social stimuli, the lights were turned off and the investigators left the room. At the end of the video, the participants were moved back into the laboratory, where they sat quietly before subsequent testing.

\section{Control treatment}

The control session was completed at the same venue, as per the other treatments, with time-matched saliva sampling and performance testing. Across all sessions, the participants remained seated to prevent any residual effects of physical movement, apart from the exercise sprints and moving to and from the video room. ${ }^{10}$ Any communication between the investigators and participants was limited to simple verbal instructions to minimise the influence of social interactions on $\mathrm{T}$ release. ${ }^{29}$ These instructions were partly scripted to ensure consistent feedback was given. The participants were also instructed to keep their food and fluid intake consistent on each testing day, ${ }^{16}$ but to avoid all forms of caffeine intake (e.g. coffee, tea, chocolate) and other ergogenic aids (e.g. energy drinks, sport supplements). Moreover, they were told to refrain from eating food and drinking fluids (except water) two hours before testing to reduce fluctuations in salivary hormones. ${ }^{30}$ The participants were further encouraged to get at least eight hours of sleep each night preceding their assessments. 


\section{Saliva collection and testing}

Steroid measurements in saliva offer the benefits of being stress free and relatively rapid to collect, with salivary steroids representing the biological active blood-free hormone. ${ }^{31}$ Saliva samples were collected by passive drool (i.e. $1 \mathrm{ml}$ over 1-2 minutes) without stimulation before each stimulus and 15 minutes afterwards to coincide with expected $\mathrm{T}$ changes in this fluid. ${ }^{13,16,18}$ The samples were stored according to published guidelines. ${ }^{32}$ The time between sample collections was $\sim 30$ minutes, once allowing for the physical tests, treatments and subsequent recovery periods. The samples were analysed in duplicate for $\mathrm{T}$ concentrations using a commercial immunoassay kit (Salimetrics LLC, USA). The T plates had a detection limit of $6.1 \mathrm{pg} / \mathrm{ml}$ with inter-assay CV's of less than $12 \%$. Samples for each participant were tested in the same assay to eliminate inter-assay variance.

\section{Physical performance testing}

Physical performance was tested immediately after each saliva collection ( $\sim 17-22$ minutes after the final sprint trial and aggressive video), taking around five minutes to complete both the HGS and CMJ PP assessments. Using a hand-held dynamometer (Takei, Japan), the HGS assessment was performed first whilst the participants were seated in an upright position. Holding the dynamometer in the dominant hand, the elbow was flexed to a 90-degree angle and each subject was instructed to apply maximal force for a period of three seconds before releasing their grip. ${ }^{33}$ Three trials were performed with 45 seconds rest between each trial. Grip strength was measured to the nearest $0.5-\mathrm{kg}$ by the lead investigator and the best trial was analysed.

The CMJ PP assessment was performed on a portable Kistler force plate (Type 92866AA, Kistler Instruments Ltd). The participants were instructed to squat down to a self-selected depth, before jumping as high as possible to reach maximal vertical height. To remove the effects of arm swing, the hands were kept on the waist during the CMJ. Three maximal trials were performed, with participants resting for 45 seconds between each trial, and the best effort was analysed. Force-time data were collected at a sampling frequency of $1000 \mathrm{~Hz}$ and CMJ PP was calculated, as described. ${ }^{34}$ Calibration of the force platform was performed before each session and the testing location was chosen to reduce potential sources of interference. Pilot testing of healthy adults $(\mathrm{n}=10)$ revealed good reliability $(\mathrm{CV}<2 \%)$ for the performance measures outlined.

\section{Statistical analyses}

All data were assessed using non-parametric statistics. The treatment responses were examined using a two-step process; first, within-session changes were calculated (post - pre presented as a percent value) and compared to zero using a Wilcoxon signed-rank test; ${ }^{10}$ second, the within-session changes were compared using a generalised estimation equation model with an autoregressive correlational structure. ${ }^{35}$ Main effects (treatment, gender) and interactions (treatment $\times$ gender) were determined by significance testing of the Wald chi-square statistic. To account for gender differences in T concentrations, HGS and CMJ PP (men $>$ women), the pre-treatment values were included as covariates. Post hoc testing was conducted using the Bonferroni sequential procedure. Relationships between the measured variables (i.e. pre-test values, relative changes) were assessed using Spearman correlations. Significance was set at $p \leq 0.05$. The results are presented as mean $\pm \mathrm{SD}$.

\section{RESULTS}

We found no significant within-treatment $\mathrm{T}$ changes in men (Table 1), whereas women had a positive and negative T response to the sprint exercise $(p=0.044)$ and video sessions ( $p=0.035)$, respectively. A treatment effect on $\mathrm{T}$ was demonstrated $(p<0.001)$, with sprint exercise producing a larger T response $(17 \%$ $\pm 29 \%, p=0.004)$ than the video $(-6.3 \% \pm 19 \%, p=$ $0.101)$ and control $(-4.8 \% \pm 23 \%, p=0.209)$ sessions $(p<0.001)$. No significant within-treatment changes in HGS were identified, but the men tended $(p=0.057)$ to exhibit a greater relative HGS response $(1.8 \% \pm$ $4.3 \%, p=0.012)$ compared to women $(-0.1 \% \pm 3.7 \%$, $p=0.901)$. A relative decrease in CMJ PP was seen in men across the video session $(p=0.027)$ and in women in the control setting $(p=0.002)$. A treatment effect on CMJ PP also emerged ( $p=0.014$ ), such that the exercise changes $(-0.1 \% \pm 4.4 \%, p=0.985)$ were different from the video $(-2.2 \% \pm 5.9 \%, p=0.016)$ and tended to differ from the control $(-2.8 \% \pm 4.4 \%$, 
$p=0.002)$ session $(p=0.054)$. The CMJ PP changes in men $(-0.2 \% \pm 3.4 \%, p=0.653)$ were also greater $(p=0.002)$ than women overall $(-3.2 \% \pm 6.1 \%, p=$ $0.002)$. No other significant main effects or interaction effects were found.

The relationships between the measured variables are outlined in Table 2. In men, pre-session T levels were negatively correlated with the relative $T$ changes after sprint exercise $(p=0.036)$ and positively related to CMJ PP before the video treatment $(p=0.018)$, both of moderate strength. Negative relationships (of moderate strength) were also identified between pre-session $\mathrm{T}$ levels in women and the relative $\mathrm{T}$ changes in the sprint exercise $(p=0.020)$, video ( $p$ $=0.030)$ and control $(p=0.054)$ sessions, although the latter outcome only approached significance. In women, the baseline measures of HGS and CMJ PP were moderately and positively related under all treatment conditions $(p \leq 0.035)$.

\section{DISCUSSION}

This study compared the effectiveness of a physi-

Table 1. Salivary testosterone and performance variables in men and women across the sprint exercise, aggressive video and control treatments. Data are presented as means $\pm \mathrm{SD}$.

\begin{tabular}{|c|c|c|c|c|c|c|c|c|c|c|}
\hline & & \multicolumn{3}{|c|}{ Sprint exercise } & \multicolumn{3}{|c|}{ Aggressive video } & \multicolumn{3}{|c|}{ Control } \\
\hline & Sample & $T(p g / m l)$ & HGS (kg) & CMJ PP (W) & $\mathrm{T}(\mathrm{pg} / \mathrm{ml})$ & HGS (kg) & CMJ PP (W) & $\mathrm{T}(\mathrm{pg} / \mathrm{ml})$ & HGS (kg) & CMJ PP (W) \\
\hline \multirow[t]{3}{*}{ Men } & Pre & $153 \pm 55$ & $54.8 \pm 7.9$ & $4238 \pm 614$ & $142 \pm 30$ & $54.6 \pm 7.3$ & $4179 \pm 670$ & $145 \pm 36$ & $55.0 \pm 8.2$ & $4128 \pm 618$ \\
\hline & Post & $180 \pm 49$ & $55.8 \pm 7.7$ & $4222 \pm 636$ & $143 \pm 39$ & $56.3 \pm 7.0$ & $4120 \pm 635$ & $148 \pm 35$ & $55.4 \pm 8.0$ & $4100 \pm 608$ \\
\hline & $\% \Delta$ & $20 \pm 34 \#$ & $1.8 \pm 4.5$ & $2.0 \pm 3.9 \# \dagger$ & $-1.2 \pm 19$ & $3.3 \pm 5.3$ & $-1.3 \pm 2.0 * \dagger$ & $2.1 \pm 21$ & $0.8 \pm 3.1$ & $-0.7 \pm 2.9 \dagger$ \\
\hline \multirow[t]{3}{*}{ Women } & Pre & $64 \pm 25$ & $32.9 \pm 5.2$ & $2353 \pm 359$ & $76 \pm 28$ & $32.0 \pm 4.7$ & $2381 \pm 348$ & $72 \pm 21$ & $32.3 \pm 4.6$ & $2394 \pm 374$ \\
\hline & Post & $72 \pm 19$ & $32.7 \pm 5.3$ & $2311 \pm 361$ & $67 \pm 20$ & $32.1 \pm 4.6$ & $2307 \pm 320$ & $64 \pm 16$ & $32.5 \pm 4.6$ & $2285 \pm 369$ \\
\hline & $\% \Delta$ & $15 \pm 26^{* \#}$ & $-0.7 \pm 4.7$ & $-1.8 \pm 4.1 \#$ & $-10.4 \pm 18^{*}$ & $0.2 \pm 3.4$ & $-3.0 \pm 8.0$ & $-10.3 \pm 23$ & $0.6 \pm 2.3$ & $-4.7 \pm 4.6^{*}$ \\
\hline
\end{tabular}

Key: T = testosterone; HGS = hand-grip strength; CMJ PP = countermovement jump peak power; $\Delta=$ change. *Significant withintreatment change $p<0.05$; "Significant changes (treatment effect) from the video and control sessions $p<0.05$; ${ }^{\dagger}$ Significant changes (gender effect) from women $p<0.05$.

Table 2. Correlations between the salivary testosterone and performance variables in men and women across the sprint exercise, aggressive video and control treatments.

\begin{tabular}{|c|c|c|c|c|c|c|c|c|c|c|c|c|c|c|c|c|}
\hline & \multirow[b]{2}{*}{ Variable } & \multicolumn{5}{|c|}{ Sprint exercise } & \multicolumn{5}{|c|}{ Aggressive video } & \multicolumn{5}{|c|}{ Control } \\
\hline & & $\mathrm{T} \% \Delta$ & HGS & $\begin{array}{c}\text { HGS } \\
\% \Delta\end{array}$ & $\begin{array}{c}\text { CMJ } \\
\text { PP }\end{array}$ & $\begin{array}{c}\text { CMJ } \\
\text { PP\% } \Delta\end{array}$ & $\mathrm{T} \% \Delta$ & HGS & $\begin{array}{c}\text { HGS } \\
\% \Delta\end{array}$ & $\begin{array}{c}\text { CMJ } \\
\text { PP }\end{array}$ & $\begin{array}{c}\text { CMJ } \\
\text { PP\% } \%\end{array}$ & $\mathrm{~T} \% \Delta$ & HGS & $\begin{array}{c}\text { HGS } \\
\% \Delta\end{array}$ & $\begin{array}{c}\text { CMJ } \\
\text { PP }\end{array}$ & $\begin{array}{c}\text { CMJ } \\
\text { PP\% } \%\end{array}$ \\
\hline \multirow[t]{5}{*}{ Men } & $\mathrm{T}$ & -0.61 & 0.01 & 0.03 & 0.52 & 0.12 & -0.11 & 0.34 & -0.20 & 0.66 & 0.13 & -0.34 & 0.14 & 0.25 & 0.15 & 0.30 \\
\hline & $\mathrm{T} \% \Delta$ & & -0.20 & -0.13 & -0.04 & -0.38 & & 0.13 & -0.04 & -0.42 & 0.21 & & -0.03 & 0.37 & 0.26 & -0.03 \\
\hline & HGS & & & -0.21 & 0.20 & 0.54 & & & -0.31 & 0.14 & 0.11 & & & -0.13 & 0.35 & -0.30 \\
\hline & $\mathrm{HGS} \% \Delta$ & & & & 0.03 & 0.13 & & & & 0.03 & 0.22 & & & & -0.10 & 0.07 \\
\hline & CMJ PP & & & & & -0.09 & & & & & -0.23 & & & & & -0.03 \\
\hline \multirow{5}{*}{ Women } & $\mathrm{T}$ & -0.61 & -0.10 & -0.09 & -0.04 & -0.39 & -0.58 & -0.36 & 0.25 & -0.19 & -0.03 & -0.53 & -0.27 & 0.31 & -0.16 & 0.07 \\
\hline & $\mathrm{T} \% \Delta$ & & -0.22 & 0.22 & -0.27 & 0.46 & & 0.49 & -0.16 & 0.50 & -0.13 & & 0.07 & -0.10 & -0.25 & 0.09 \\
\hline & HGS & & & -0.09 & 0.57 & 0.32 & & & -0.13 & 0.62 & -0.17 & & & -0.23 & 0.70 & 0.30 \\
\hline & $\mathrm{HGS} \% \Delta$ & & & & -0.24 & 0.32 & & & & -0.13 & -0.02 & & & & -0.38 & 0.08 \\
\hline & CMJ PP & & & & & -0.16 & & & & & -0.45 & & & & & -0.26 \\
\hline
\end{tabular}

Key: $\mathrm{T}=$ testosterone; $\mathrm{HGS}=$ hand-grip strength; $\mathrm{CMJ} \mathrm{PP}=$ countermovement jump peak power; $\Delta=$ change.

Significant correlations are highlighted in bold $p<0.05$. 
cal and psychological stimulus as practical tools for acutely modifying T release and physical performance in a healthy cohort of men and women. Overall, the $\mathrm{T}$ (increase) and CMJ PP (no change) responses to sprint exercise were superior to the respective changes induced by the aggressive video (no change, decrease) and control (no change, decrease) treatments. We also found that pre-test $\mathrm{T}$ levels were negatively related to the relative $\mathrm{T}$ changes that occurred in one or more treatments, but no variables were associated with the relative changes in HGS and CMJ PP.

Confirming our initial hypothesis, a brief bout of sprint cycling exercise promoted a T elevation (17.5\%) in healthy adults and this $\mathrm{T}$ response differed from that observed across the aggressive video (-6.3\%) and control (-4.8\%) treatments. The exercise-induced changes in $\mathrm{T}$ are likely to be regulated by several factors such as cycling cadence, ${ }^{36}$ heart rate, ${ }^{37}$ and perceived exertion. ${ }^{37}$ Whilst sprint cycling exercise can reliably increase $\mathrm{T}$ concentrations in untrained, active ${ }^{10,18,38}$ or highly-trained men, ${ }^{10,11,36}$ women do not always exhibit a T response..$^{21,27}$ This disparity could be explained by differences in the cycling protocols employed (e.g. exercise duration, load and recovery periods) and the sampling procedures (e.g. biological media, timing and frequency). Nevertheless, the positive T responses of women in this work are consistent with that achieved by other exercise ${ }^{19-21}$ and similar to male populations when expressed relative to baseline concentrations. ${ }^{15,23}$ So despite the women tested having less than half the amount of salivary $\mathrm{T}$ available as males, it appears that women can still access similar relative amounts of $\mathrm{T}$ with an appropriate physical stimulus.

The maintenance in CMJ PP (-0.1\%) following sprint exercise, in relation to the decline in the video $(-2.2 \%)$ and control $(-2.8 \%)$ treatments, could be partly due to the increase in $\mathrm{T}$ availability. Studies have demonstrated the benefits of using exercise (including high-intensity sprint cycling) to elevate $\mathrm{T}$ and improve physical performance in men. ${ }^{5,6,11}$ There is evidence linking $\mathrm{T}$ to various performance outcomes via a rapid behavioural mechanism (e.g. motivation, mood, competitive drive), ${ }^{1,16,39,40}$ which could afford some functional benefits within a short time span, as seen in this work. Alternatively, the T response might reflect improvements in mood or motivational state arising from perceptions of better performance, or increased alertness. Given the study design, we do acknowledge that the physical stimulus effect could involve other peripheral mechanisms (e.g. calcium sensitivity, preferential motor unit recruitment $)^{4}$ that were not assessed. It is also possible that the CMJ PP results were driven by a warm-up response, as the other treatments were passive in nature, and the performance effect was specific to those muscle groups exercised when cycling.

The aggressive video was less effective as a practical stimulus for increasing $\mathrm{T}$ and/or improving performance. For men, the lack of a $\mathrm{T}$ response might reflect their recreational status and the situational content of the video, being rugby based and showing elite athletes. ${ }^{10}$ Conversely, the $\mathrm{T}$ decline in women could be due to their perception of this footage as being either stressful 13 or sad. ${ }^{16}$ As further evidence of the importance of the visual content and group affiliation to it, viewing video images showing reallife aggression and violence can lead to elevations in male and female $\mathrm{T}$ levels. ${ }^{15}$ The $\mathrm{T}$ responses of the women tested herein were however similar to the control session, so the observed change might also reflect normal biological variation. Other potential factors influencing the $\mathrm{T}$ responses to psychological stress include affective state (e.g. mood) and stress hormones (e.g. pre-test cortisol, cortisol change). ${ }^{15,22,26}$ The video treatment also lowered CMJ PP by $1.3 \%$ in men only, but the magnitude of change was smaller than the measurement error for this assessment.

On an individual level, those adults with higher pre-test $\mathrm{T}$ levels tended to produce smaller $\mathrm{T}$ responses under physical and/or psychological stress, as demonstrated elsewhere. ${ }^{26,41} \mathrm{We}$ also found greater consistency in these relationships in women under all treatment conditions. This finding may be the result of having lower circulating $\mathrm{T}$ levels and different $\mathrm{T}$ sources in females (i.e. ovaries, conversion of adrenal androgens) relative to males (i.e. gonadal)., ${ }^{1,27}$ Despite the perceived importance of $\mathrm{T}$, the baseline and reactive $\mathrm{T}$ measures taken did not correlate with the relative changes in HGS or CMJ PP. This is perhaps not surprising, as these associations tend to be stronger and more consistent among elite strength- 
trained cohorts, ${ }^{1,39}$ who arguably possess a more developed neuromuscular system and better linkage with other physiological (e.g. hormonal) systems. ${ }^{1}$ This viewpoint is partly supported by the fact that performance either decreased or did not change in this study, whilst similar sprint exercise and video treatments resulted in improvements in strength ${ }^{5,16}$ and power ${ }^{11}$ in highly-trained populations, with additional correlations between the hormonal and performance outcomes. ${ }^{5,16}$

In conclusion, a short bout of sprint exercise promoted a positive $\mathrm{T}$ response in healthy adults and maintained CMJ PP, relative to the video and control treatments. Those men and women with higher pre-test $\mathrm{T}$ levels also tended to produce smaller $\mathrm{T}$ responses to one or more treatments, but no variables correlated with the changes in physical performance. These data highlight the importance of stimulus selection and individual predispositions when attempting to acutely modify $\mathrm{T}$ and physical performance in healthy adults.

\section{ACKNOWLEDGEMENTS}

The authors would like to thank the individuals who participated in this study.

\section{CONFLICT OF INTEREST}

The authors do not have any conflict of interest.

\section{FINANCIAL SUPPORT}

This project was undertaken as part of the Elite Sport Performance Research in Training (ESPRIT) with Pervasive Sensing Programme [EP/H009744/1], funded by the Engineering and Physical Sciences Research Council, UK, and UK Sport.

\section{REFERENCES}

1. Crewther BT, Cook C, Cardinale M, Weatherby RP, Lowe T, 2011 Two emerging concepts for elite athletes: The short-term effects of testosterone and cortisol on the neuromuscular system and the dose-response training role of these endogenous hormones. Sports Med 41: 103-23.

2. Vingren JL, Kraemer WJ, Ratamess NA, et al, 2010 Testosterone physiology in resistance exercise and training: The up-stream regulatory elements. Sports
Med 40: 1037-53.

3. West DW, Burd NA, Staples AW, Phillips SM, 2010 Human exercise-mediated skeletal muscle hypertrophy is an intrinsic process. Int J Biochem Cell B 42: 13711375.

4. Kilduff LP, Finn CV, Baker JS, Cook CJ, West DJ, 2013 Preconditioning strategies to enhance physical performance on the day of competition. Int J Sports Physiol Perform 8: 677-681.

5. Crewther BT, Cook CJ, Lowe TE, Weatherby RP, Gill N, 2011 The effects of short cycle sprints on power, strength and salivary hormones in elite rugby players. J Strength Cond Res 25: 32-39.

6. Cook CJ, Kilduff LP, Crewther BT, Beaven M, West DJ, 2014 Morning based strength training improves afternoon physical performance in rugby union players. J Sci Med Sport 17: 317-321.

7. Rønnestad BR, Nygaard H, Raastad T, 2011 Physiological elevation of endogenous hormones results in superior strength training adaptation. Eur J Appl Physiol: 111: 2249-2259.

8. Hansen S, Kvorning T, Kjaer M, Sjøgaard G, 2001 The effect of short-term strength training on human skeletal muscle: the importance of physiologically elevated hormone levels. Scand J Med Sci Sport 11: 347-354.

9. Beaven MC, Cook CJ, Gill ND, 2008 Significant strength gains observed in rugby players after specific resistance exercise protocols based on individual salivary testosterone responses. J Strength Cond Res 22: 419-425.

10. Crewther BT, Kilduff LP, Cook CJ, 2014 Trained and untrained males show reliable salivary testosterone responses to a physical stimulus, but not a psychological stimulus J Endocrinol Invest 37: 1065-1072.

11. Obmiński Z, Borkowski L, Ladyga M, Hübner-Woźniak E, 1998 Concentrations of cortisol, testosterone and lactate, and power output in repeated, supramaximal exercise in elite fencers. Biol Sport 15: 19-24.

12. Goto K, Ishii N, Kurokawa K, Takamatsu K, 2007 Attenuated growth hormone response to resistance exercise with prior sprint exercise. Med Sci Sports Exerc 39: 108-115.

13. Hellhammer DH, Hubert W, Schürmeyer T, 1985 Changes in saliva testosterone after psychological stimulation in men. Psychoneuroendocrino 10: 77-81.

14. Stoléru SG, Ennaji A, Cournot A, Spira A, 1993 LH pulsatile secretion and testosterone blood levels are influenced by sexual arousal in human males. Psychoneuroendocrino 18: 205-218.

15. Fukui H, Yamashita M, 2003 The effects of music and visual stress on testosterone and cortisol in men and women. Neuroendocrinol Lett 24: 173-180.

16. Cook CJ, Crewther BT, 2012 Changes in salivary testosterone concentrations and subsequent voluntary squat performance following the presentation of short video clips. Horm Behav 61: 17-22.

17. Kilduff LP, Hopp RN, Cook CJ, Crewther BT, Man- 
ning JT, 2013 Digit ratio (2D:4D), aggression, and testosterone in men exposed to an aggressive visual stimulus. Evol Psychol 11: 953-964.

18. Beaven MC, Ingram JR, Gill ND, Hopkins WG, 2010 Ultradian rhythmicity and induced changes in salivary testosterone. Eur J Appl Physiol 110: 405-413.

19. Nindl BC, Kraemer WJ, Gotshalk LA, et al, 2001 Testosterone responses after resistance exercise in women: influence of regional fat distribution. Int J Sport Nutr Exerc Metab 11: 451-465.

20. Kochańska-Dziurowicz A, Gawel-Szostek V, Gabryś T, Kmita D, 2001 Changes in prolactin and testosterone levels induced by acute physical exertion in young female athletes. Fiziologiya Cheloveka 27: 100-103.

21. Enea C, Boisseau N, Ottavy M, et al, 2009 Effects of menstrual cycle, oral contraception, and training on exercise-induced changes in circulating DHEA-sulphate and testosterone in young women. Eur J Appl Physiol 106: 365-373.

22. Lopez HH, Hay AC, Conklin PH, 2009 Attractive men induce testosterone and cortisol release in women. Horm Behav 56: 84-92.

23. Jiménez M, Aguilar R, Alvero-Cruz JR, 2012 Effects of victory and defeat on testosterone and cortisol response to competition: Evidence for same response patterns in men and women. Psychoneuroendocrino 37: 15771581 .

24. Okudan N, Gökbel H, Uçok K, Baltaci AK, 2005 Serum leptin concentration and anaerobic performance do not change during the menstrual cycle of young females. Neuroendocrinol Lett 26: 297-300.

25. Bird SP, Tarpenning KM, 2004 Influence of circadian time structure on acute hormonal responses to a single bout of heavy-resistance exercise in weight-trained men. Chronobiol Int 21: 131-146.

26. Maestripieri D, Baran NM, Sapienza P, Zingales L, 2010 Between- and within-sex variation in hormonal responses to psychological stress in a large sample of college students. Stress 13: 413-424.

27. Thomas NE, Leyshon A, Hughes MG, et al, 2010 Concentrations of salivary testosterone, cortisol, and immunoglobulin A after supra-maximal exercise in female adolescents. J Sports Sci 28: 1361-1368.

28. Millet K, Dewitte S, 2007 Digit ratio (2D:4D) moderates the impact of an aggressive music video on aggression. Pers Indiv Differ 43: 289-294.
29. DeSoto CM, Hitlan RT, Deol RS, McAdams D, 2009 Testosterone fluctuations in young men: The difference between interacting with like and not-like others. Evol Psychol 8: 173-88.

30. Gibson LE, Checkley S, Papadopoulos A, et al, 1999 Increased salivary cortisol reliable induced by a proteinrich midday meal. Psychosom Med 61: 214-224.

31. Arregger AL, Contreras LN, Tumilasci OR, Aquilano DR, Cardoso EML, 2007 Salivary testosterone: a reliable approach to the diagnosis of male hypogonadism. Clin Endocrinol 67: 656-662.

32. Toone RJ, Peacock OJ, Smith AA, et al, 2013 Measurement of steroid hormones in saliva: Effects of sample storage condition. Scand J Clin Lab Invest 73: 615-621.

33. Leyk D, Gorges W, Ridder D, et al, 2007 Hand-grip strength of young men, women and highly trained female athletes. Eur J Appl Physiol 99: 415-421.

34. West DJ, Owen NJ, Jones MR, et al, 2011 Relationships between force-time characteristics of the isometric midthigh pull and dynamic performance in professional rugby league players. J Strength Cond Res 25: 3070-3075.

35. Liang K-Y, Zeger SL, 1986 Longitudinal data analysis using generalized linear models. Biometrika 73: 13-22.

36. Smith AA, Toone R, Peacock O, et al, 2013 Dihydrotestosterone is elevated following sprint exercise in healthy young men. J Appl Physiol 114: 1435-1440.

37. Copeland JL, Consitt LA, Tremblay MS, 2002 Hormonal responses to endurance and resistance exercise in females aged 19-69 years. J Geront Series A: Biol Sci Med Sci 57A: B158-165.

38. Goto K, Ishii N, Kizuka T, et al, 2009 Hormonal and metabolic responses to slow movement resistance exercise with different durations of concentric and eccentric actions. Eur J Appl Physiol 106: 731-739.

39. Crewther BT, Cook CJ, Gaviglio CM, Kilduff LP, Drawer S, 2012 Baseline strength can influence the ability of salivary free testosterone to predict squat and sprinting performance. J Strength Cond Res 26: 261-268.

40. Cook CJ, Beaven MC, 2013 Salivary testosterone is related to self-selected training load in elite female athletes. Physiol Behav 116-117: 8-12.

41. Zilioli S, Watson NV, 2013 Winning isn't everything: mood and testosterone regulate the cortisol response in competition. PLoS One 8: e52582. 